\title{
POLARITY IN A COMPLETELY DISTRIBUTIVE COMPLETE LATTICE
}

\author{
ZIKE DENG
}

(Communicated by William C. Waterhouse)

\begin{abstract}
We introduce $p$-bases in completely distributive complete polarity lattices and give a procedure for generating these lattices by $p$-bases.
\end{abstract}

1. Introduction. As in [1], the object of this paper is to lay a lattice-theoretic foundation of treating local properties of the generalized algebra of topology i.e., the algebra of topology [3] with polarity instead of Boolean complement. $p^{\sim}$ in Definition 3.2 is introduced to overcome the difficulty in the unified treatment of Boolean complement and non-Boolean polarity and Proposition 3.2 can be regarded as a generalization of Boolean property.

We first examine polarity by means of quasi-atoms introduced in [1]. And then we introduce $p$-bases in completely distributive complete polarity lattices and give a procedure for the generation of these lattices by $p$-bases.

2. Preliminaries. Let $(L, \leq)$ be a completely distributive complete lattice. We shall use $a, b, c$ to denote its elements and $A, B, C$ its subsets.

Definition 2.1. $a \prec b$ iff for every $A$ with $\bigvee A=b$ there exists $c \in A$ such that $a \leq c$.

DEFINITION $2.2[1] . p \in \Delta(L) \subseteq L$ where $\Delta(L)=\left\{\bigwedge_{a \prec b} b \mid a \neq 0, \bigvee_{a \ddagger c} C<1\right\}$ is called a quasi-atom.

We shall use $p, q, r$ to denote elements of $\Delta(L)$ and $P, Q, R$ its subsets.

DEFINITION $2.3[\mathbf{1}]$. (1) $Q \subseteq \Delta(L)$ is called a base of $(L, \leq)$ iff $a=\bigvee\{p \mid p \in$ $Q, p \prec a\}$ for every $a \in L$.

(2) $p \in \Delta(L)$ has the $\wedge$-property iff for every $a, b \in L$, that $p \prec a$ and $p \prec b$ implies $p \prec a \wedge b$.

(3) A base $Q$ of which every element is $\vee$-irreducible and has the $\wedge$-property is called a $s$-base.

(4) $(L, \leq)$ is standard iff it has a $s$-base.

(5) A $l$-set of a base $Q$ is a subset $R$ of $Q$ satisfying (1) $p \in R$ implies that there exists $q$ such that $p \prec q \in R$ and (2) $p \prec q \in R$ implies $p \in R$ for every $p \in Q$.

(6) For $a \in L, R_{a}=\{p \mid p \prec a, p \in Q\}$.

The set of all $l$-sets of a base $Q$ will be denoted by $L(Q)$. Evidently $R_{a} \in L(Q)$ for every $a \in L$. Let $\Phi: Q \rightarrow L(Q)$ be defined as $\Phi(p)=R_{p}$. We shall use $I(L)$ to denote the set of all completely $\vee$-irreducible elements of $L$ and $A(L)$ the set of all its atoms.

Received by the editors November 3, 1986 and, in revised form, April 23, 1987.

1980 Mathematics Subject (lassification (1985 Revision). Primary 06D10, 06D30.

Key words and phrases. Quasi-atoms, $p$-bases. 


\section{Polarity.}

DEFINITION 3.1 [2]. A polarity' in a lattice is involutary antiautomorphism.

Throughout the remaining part of this paper $\left(L, \leq,^{\prime}\right)$ will denote a completely distributive complete polarity lattice (in short $p$-lattice). By a Boolean $p$-lattice we mean a $p$-lattice with Boolean complement as polarity.

DEFINITION 3.2. $p^{\sim}=\bigwedge_{q \nless p^{\prime}} q$ for $p, q \in \Delta(L)$.

LEMMA 3.1. $q \prec p^{\prime}$ implies $p \prec q^{\prime}$ for every $p, q \in \Delta(L)$.

PROOF. By [1, Proposition 2.1] $p=\bigwedge_{p \prec a} a$, hence $p^{\prime}=\bigvee_{p \prec a} a^{\prime}$. Since $q \prec p^{\prime}$ implies that there exists $a$ with $p \prec a$ such that $q \leq a^{\prime}$, it follows that $p \prec q^{\prime}$ by $[\mathbf{1}$, Lemma 2.1(5)]. Q.E.D.

LEMMA 3.2. $p^{\sim} \in \Delta(L)$.

PROOF. Since $p \neq 0$, there exists $r \in \Delta(L)$ such that $r \prec p^{\prime}$ by $[\mathbf{1}$, Lemma $3.2(2)$ ] noting that $\Delta(L)$ itself is a base. It follows that $p^{\sim} \leq r$, which implies $\bigvee_{p \sim \ddagger a} a \leq \bigvee_{r \ddagger b} b<1$. By Lemma $3.1 p^{\sim^{\prime}}=\bigvee_{p \nprec q^{\prime}} q^{\prime}$, so $p \nprec p^{\sim^{\prime}}$, hence $p^{\sim} \neq 0$. Since $\bigwedge_{p^{\sim} \prec a} a \leq \bigwedge_{q \nless p^{\prime}} \bigwedge_{q \prec b} b=\bigwedge_{q \nless p^{\prime}} q$ by $\left[1\right.$, Proposition 2.1], $p^{\sim}=\bigwedge_{p^{\sim} \prec a} a \in$ $\Delta(L)$. Q.E.D.

Proposition 3.1. $p^{\sim} \nprec p^{\prime}$ and for every $q \in \Delta(L), q \nprec p^{\prime}$ implies $p^{\sim} \leq q$.

PROOF. In the proof of Lemma 3.2 we have already shown that $p \nprec p^{\sim \prime}$ and so $p^{\sim} \nprec p^{\prime}$ by Lemma 3.1. The remaining part is trivial. Q.E.D.

PROPOSITION 3.2. $p\left\lfloor a^{\prime}\right.$ iff $p^{\sim} \prec a$ for every $p \in \Delta(L), a \in L$.

ProOF. $p \Varangle a^{\prime}$ iff $a \nless p^{\prime}$, which holds iff there exists $q \in \Delta(L)$ such that $q \prec a$ and $q \nprec p^{\prime}$ by [1, Lemma 3.2(2)], which holds iff $p^{\sim} \prec a$ by Proposition 3.1 and [1, Lemma 2.1(2), Lemma 3.2(1)]. Q.E.D.

Proposition 3.3. (1) $p^{\sim \sim}=p$. (2) $p \prec q$ implies $q^{\sim} \prec p^{\sim}$.

ProOF. (1) Since $p^{\sim} \nprec p^{\prime}, p \nprec p^{\sim \prime}$, which implies $p^{\sim} \leq p$. Futhermore $p^{\sim \sim} \nprec p^{\sim \prime}$, so $p^{\sim} \nprec p^{\sim \sim 1}$, hence $p \leq p^{\sim \sim}$.

(2) Suppose $p \prec q$, then $p^{\sim} \nless q^{\prime}$, hence $q \Varangle p^{\sim \prime}$ and so $q^{\sim} \prec p^{\sim}$. Q.E.D.

From above we see that $p^{\sim}=\min \left\{q \mid q \nprec p^{\prime}, q \in \Delta(L)\right\}$ and $\sim: \Delta(L) \rightarrow \Delta(L)$ is a mapping satisfying Propositions 3.2 and 3.3. If $\left(L, \leq_{,}^{\prime}\right)$ is a Boolean $p$-lattice, then $\Delta(L)=A(L)$, the binary relation $\prec$ is the same as $\leq$ and $p^{\sim}=p$. We shall need the following lemmas.

LEMMA 3.3. $p \in \Delta(L)$ is $\vee$-irreducible iff $p^{\sim}$ has the $\wedge$-property.

PROOF. For $p \leq a \vee b$ iff $p^{\sim} \nprec a^{\prime} \wedge b^{\prime}$. Q.E.D.

LEMMA 3.4. If $\sim: Q \rightarrow Q$ where $Q \subseteq \Delta(L)$ satisfies Proposition 3.2 and Proposition 3.3(1), then $p^{\sim}=\min \left\{q \mid q \nprec p^{\prime}, q \in \Delta(L)\right\}$.

PROOF. Since $p \leq p$, it follows that $p^{\sim} \nprec p^{\prime}$. On the other hand if $q \in \Delta(L)$ and $q \nprec p^{\prime}$, then $p \nprec q^{\prime}$, hence $p^{\sim} \leq q$. Q.E.D.

4. $p$-bases.

DEFINITION 4.1. A $p$-base of $\left(L, \leq,^{\prime}\right)$ is a base $Q$ which is closed under .

PROPOSITION 4.1. Any p-lattice has a $p$-base.

ProOF. By [1, Proposition 2.2] $\Delta(L)$ itself is a base and $p$-base. Q.E.D. 
PROPOSITION 4.2. (1) Any standard p-lattice has a p-s-base.

(2) Any $p$-lattice isomorphic to a complete ring of sets has a p-base $I(L)$.

(3) Any Boolean p-lattice has a p-base $A(L)$.

PROOF. (1) $Q=\{p \mid p \in \Delta(L), p$ is $\vee$-irreducible and $p$ has the $\wedge$-property $\}$ is evidently a $s$-base and by Lemma 3.3 and Proposition 3.3(1) a $p$-base.

(2) By Proposition 3.3 (2) $p \prec p$ implies $p^{\sim} \prec p^{\sim}$ and so $p^{\sim} \in I(L)$ by [1, Lemma 2.1(4)].

(3) For $p^{\sim}=p \in A(L)$. Q.E.D.

For $R_{p}, L(P)$ and $\Phi$ in the following propositions see $\S 2$ of this paper.

PROPOSITION 4.3. If $Q$ is a p-base, then

(1) $\prec_{Q} \circ \prec_{Q}=\prec_{Q}$ where $\prec_{Q}$ is the restriction of $\prec$ on $Q$.

(2) For every $p, q, r, s \in Q, q \prec r \in \bigcap_{p \prec s} R_{s}$ implies $q \prec p$.

(3) For every $p \in Q$ there exists $q, r \in Q$ such that $q \prec p \prec r$.

(4) For every $p, q \in Q, R_{p}=R_{q}$ implies $p=q$.

(5) For every $p, q, r \in Q$, that $R_{p} \subseteq R_{q}$ and $q \prec r$ implies $p \prec r$.

(6) For every $p, q \in Q, p \prec q$ implies $q^{\sim} \prec p^{\sim}$.

(7) For every $p, p^{\sim \sim}=p$.

If $Q$ is a $p$-s-base, then

(8) For every $p, q, r \in Q, p \in R_{q} \cap R_{r}$ implies that there exists $s \in Q$ such that $p \prec s$ and $s \in R_{q} \cap R_{r}$.

(9) $R_{p}$ is $\vee$-irreducible in $L(Q)$ ordered by inclusion.

ProOF. It follows from [1, Proposition 3.3] and Proposition 3.3. Q.E.D.

Let $P_{i, j}, \ldots$ be any nonvoid set on which there are defined a binary relation $\prec$ and a mapping $\sim$ satisfying the conditions $(i),(j), \ldots$ in Proposition 4.3 .

PROPOSITION 4.4. (1) $L\left(P_{1,2,6,7}\right)$ ordered by inclusion is a p-lattice.

(2) $\Phi\left(P_{1,2, \ldots, 7}\right)$ is a $p$-base of $L\left(P_{1,2, \ldots, 7}\right)$ and $\Phi$ is an isomorphism from $P_{1,2, \ldots, 7}$ onto $\Phi\left(P_{1,2, \ldots, 7}\right)$.

(3) $\Phi\left(P_{1,2, \ldots, 9}\right)$ is a $p$-s-base.

PrOOF. (1) By [1, Proposition 4.1(1)] $L\left(P_{1,2,6,7}\right)$ is a completely distributive complete lattice. Define ' as follows: $R^{\prime}=\{p \mid$ there exists $q$ such that $p \prec q$ and $\left.q^{\sim} \notin R\right\}$ for $R \in L\left(P_{1,2,6,7}\right)$ and $p, q \in P_{1,2,6,7}$. That $R \leq S$ implies $S^{\prime} \leq R^{\prime}$ is trivial. Since $p \in R$ iff there exists $q$ such that $p \prec q$ and $R_{q} \subseteq R$, and since $R_{q} \subseteq R$ iff for every $s, q^{\sim} \prec s$ implies $s^{\sim} \in R$, which is equivalent to $q^{\sim} \notin R^{\prime}$, it follows that $p \in R$ is equivalent to $p \in R^{\prime \prime}$, hence $R^{\prime \prime}=R$. Thus ' is a polarity.

(2) By [1, Proposition 4.1(1)-(4)] $\Phi\left(P_{1,2, \ldots, 7}\right)$ is a base, $\Phi$ is a bijection and both $\Phi$ and $\Phi^{-1}$ are $\prec$-preserving. Define $R_{p}^{\sim}=R_{p^{\sim}}$. Then $R_{p} \prec S^{\prime}$ iff $p \in S^{\prime}$, which holds iff there exists $q$ such that $p \prec q$ and $q^{\sim} \notin S$, which holds iff there exists $q^{\sim}$ such that $q^{\sim} \prec p^{\sim}$ and $q^{\sim} \notin S$, which is equivalent to $R_{p}^{\sim}=R_{p} \sim \not S$. Evidently $R_{p}^{\sim} \sim=R_{p}$. By Lemma $3.4 R_{p}^{\sim}=\min \left\{R \mid R \nprec R_{p}^{\prime}, R \in \Delta\left(L\left(P_{1,2, \ldots, 7}\right)\right)\right\}$ and so $\Phi\left(P_{1,2, \ldots, 7}\right)$ is a $p$-base. Since $\Phi\left(p^{\sim}\right)=R_{p^{\sim}}=R_{p}^{\sim}=\Phi(p)^{\sim} \Phi$ is an isomorphism.

(3) By [1, Proposition 4.1(5),(6)] and (2) $\Phi\left(P_{1,2, \ldots, 9}\right)$ is a $p$-s-base. Q.E.D.

The above proposition can be used to construct new $p$-lattices from old ones. 


\section{REFERENCES}

1. Z. Deng, Quasi-atoms and complete distributivity, Proc. Amer. Math. Soc. 103 (1988), 365-370

2. M. Kamara, Zur konstraktion Vollstandiger Polaritätsverbande, J. Reine Angew. Math. 299/300 (1978), 280-289.

3. J. C. C. McKinsey and A. Tarski, The algebra of topology, Ann. of Math. (2) 45(1944), 141191.

Department of Applied Mathematics, Hunan University, Changsha, hunan, China 